\title{
Maßnahmenvorschläge zur Erhöhung der Biodiversität von Arthropoden in Erwerbsobstanlagen
}

\author{
Doris Dannenmann ${ }^{1} \cdot$ Elke Hietel $^{1} \cdot$ Thomas Wagner $^{2}$ \\ Eingegangen: 26. Januar 2021 / Angenommen: 2. Juli 2021 / Online publiziert: 23. Juli 2021 \\ (c) Der/die Autor(en) 2021
}

\section{Zusammenfassung}

Gerade Dauerkulturen, welche über viele Jahre bestehen bleiben, können einen großen ökologischen Wert für Insekten und Spinnentiere darstellen, wenn die negativen Einflüsse durch die Bewirtschaftung durch gezielte Maßnahmen minimiert werden. Im Rahmen eines Forschungsprojektes wurde von 2016 bis 2019 an der TH Bingen in Zusammenarbeit mit der Gemeinde Zornheim (Rheinhessen), zwei dort ansässigen Obstbauern, dem Dienstleistungszentrum Ländlicher Raum Rheinhessen-Nahe-Hunsrück (DLR RNH) und der Universität Koblenz-Landau (Campus Koblenz), gefördert durch die Stiftung Natur- und Umwelt Rheinland-Pfalz die Biodiversität von Obstanlagen untersucht. Es wurde eine intensive Erfassung der Insekten und Spinnentiere auf Basis von Individuenzahlen, Verteilung der Tiere auf Großgruppen, Artenzahlen der Käfer (inkl. Rote Liste) und Wanzen, deren Diversität und der sie beeinflussenden Faktoren auf vier Erwerbsobstflächen und einer Streuobstwiese als Referenzfläche untersucht. Mittels eines im Projekt erarbeiteten Bewertungsbogens, wurden die Auswirkungen der fünf Faktorenkomplexe: Sonderstrukturen, Anlagenstruktur, Beschattung, Mahdregime und Einsatz von Pflanzenschutzmitteln erhoben. Die visuelle Darstellung der Einflussfaktoren mittels Netzdiagramm zeigt biodiversitätsbeeinträchtigende Faktoren und ermöglicht so Empfehlungen zur Aufwertung der Biodiversität. Die hier vorgeschlagenen Maßnahmen zielen auf eine Erhöhung der Biodiversität und Minimierung der negativen Auswirkungen der Bewirtschaftung in Erwerbsobstanlagen. Durch eine möglichst große, an die Kultur angepasste Strukturvielfalt soll die Verfügbarkeit von Nistplätzen, Nahrung und Überwinterungsmöglichkeiten für Insekten und Spinnentiere deutlich erhöht werden und damit zur Ausbildung stabiler Populationen führt.

Schlüsselwörter Biodiversität · Erwerbsobstanlagen · Insekten · Spinnen · Obstbau

Doris Dannenmann

d.dannenmann@th-bingen.de

Elke Hietel

e.hietel@th-bingen.de

Thomas Wagner

thwagner@uni-koblenz.de

1 Hermann-Hoepke-Institut, Technische Hochschule Bingen,

Berlinstr. 109, 55411 Bingen, Deutschland

2 Institut für integrierte Naturwissenschaften - Biologie,

Universität Koblenz-Landau, Campus Koblenz,

Universitätstr. 1, 56070 Koblenz, Deutschland 


\title{
Suggested measures to increase biodiversity of arthropods in orchards
}

\begin{abstract}
Cultured orchards, that remain for many years, are able to become a great value for insects and spiders, if the negative influences of cultivation will be minimized by specific measures.

In our project we made on four integrated cultured orchards and one orchard meadow as reference an intensive recording of the insects and arachnids, based on individual numbers, distribution to large groups, number of species (beetles, bugs), their biodiversity (Shannon), Red Data Book species and their influencing factors. By an evaluation form, created in project, the effects of five clusters of factors: separate structures, orchard structures, shading, mowing and application of pesticides. The visual presentation of the influencing factors with star-plot shows restricting effects on biodiversity and offers possibilities to rise the biodiversity. The proposed measures are aimed at increasing the biodiversity and decrease the negative effects of cultivation in cultured orchards. The largest structural diversity, adapted to cultivation, increases the availability of nesting sites, food, and wintering quarters for insects and spiders significant and develop stable populations.
\end{abstract}

Keywords Biodiversity $\cdot$ Commercial orchards $\cdot$ Insects $\cdot$ Spiders $\cdot$ Fruit growing

\section{Einleitung}

Obstanlagen als Dauerkultur können eine hohe ökologische Bedeutung für Flora und Fauna erreichen. Sie bieten über viele Jahre einen vielfältigen Lebensraum. Neben dem Grünland, welches jahrelang nicht umgebrochen wird, stehen als weitere Strukturen Bäume mit Lebensräumen in und am Holz (z. B. Rinde, Höhlen, Mulm, Totholz), im Laub und Blüten als Nahrung zur Verfügung. Für Streuobstwiesen ist die hohe ökologische Bedeutung bereits nachgewiesen worden (Landesamt für Umweltschutz 1992; Achtziger et al. 1999). Da es sich in den vergangenen Jahrzehnten als schwierig erwiesen hat, Streuobstwiesen in einem geeigneten Pflegezustand zu halten und die Flächen ebenso wie der Erwerbsobstanbau weiter zurückgehen (Statistisches Landesamt RLP 2012, 2017), stellt sich die Frage, welche ökologische Bedeutung in der intensiven Nutzung stehende Obstanlagen haben und wie man vorhandenes Potenzial erhöhen kann.

In den Jahren 2016 bis 2018 wurde an der TH Bingen in Zusammenarbeit mit der Gemeinde Zornheim (Rheinhessen), zwei dort ansässigen Obstbauern, dem Dienstleistungszentrum Ländlicher Raum Rheinhessen-Nahe-Hunsrück (DLR RNH) und der Universität in Koblenz, gefördert durch die Stiftung Natur- und Umwelt Rheinland-Pfalz im Rahmen eines Forschungsprojektes die Biodiversität von Obstanlagen untersucht.

\section{Untersuchungsgebiet}

Die Untersuchungsflächen liegen in Zornheim, im Kreis Mainz-Bingen (Abb. 1). Die bevorzugte Lage im milden, trockenen Klima Rheinhessens begünstigt hier seit Jahrhunderten den Obst- und Weinbau.
Im Untersuchungsgebiet (Abb. 2) wurden in Abstimmung mit den ansässigen Obstanbauern fünf mit Obstbäumen bestandene Flächen (Abb. 3) ausgewählt. Um ein möglichst großes Spektrum an Obstanlagen (Erwerbsobstanbau mit Niederstämmen) zu erhalten, wurden untersucht:

- eine Apfelanlage mittleren Alters (Abb. 3a), Größe: 17 ha, Pflanzjahr 2002, engstehende Spindelbäume, glatte Rinde, kleine lichte Krone, wenig Beschattung, kein Moos- und Flechtenbewuchs, Baumstreifen durch Herbizid freigehalten (2/a), Fahrgasse häufig gemulcht (zwölf Mal pro Jahr), sehr kurz gehalten, 29 Fungizid- und Blattdüngungseinsätze, sechs Insektizideinsätze (2016).

- eine junge Kirschanlage (Abb. 3b), Größe: 13ha, Pflanzjahr 2013, relativ hohe (>1 m) noch dünne, glatte Stämme, lichte Kronen, geringe Beschattung, Baumstreifen durch Herbizid freigehalten (2/a), Fahrgasse teilweise länger (fünf Mulchgänge, zwei Drittel der Gasse), zehn Fungizid- und sonstiger Mitteleinsatz, fünf Insektizidapplikationen (2016).

- eine alte Birnenanlage (Abb. 3c), Größe: 0,5 ha, Pflanzjahr 1969, mittlere Stämme, raue Rinde, kaum Totholz, schmale Kronen, leichter Moos- und Flechtenbewuchs, Baumstreifen mit Herbizid freigehalten (1/a), Fahrgasse teilweise länger (vier Mulchgänge/a), 21 Fungizideinsätze, sieben Insektizidapplikationen (2016).

- eine alte Mirabellenanlage (Abb. 3d) Größe: 8ha, Pflanzjahr 1969, alte Bäume mit kräftigen Stämmen (Viertelstamm), rauer Rinde, Totholzanteile am Baum mit Höhlenbildung, große, dichte Kronen dadurch starke Beschattung und starker Moos- und Flechtenbewuchs, Baumstreifen mit Herbizid freigehalten (2/a), Bewuchs in Fahrgasse teilweise länger (sechs Mulchgänge/a, jeweils nur zwei Drittel der Fahrgasse), acht Fungizideinsätze, sechs Insektizidapplikationen (2016). 
Abb. 1 Karte Zornheim südlich von Mainz, RLP. (Quelle: Karten, Apple)

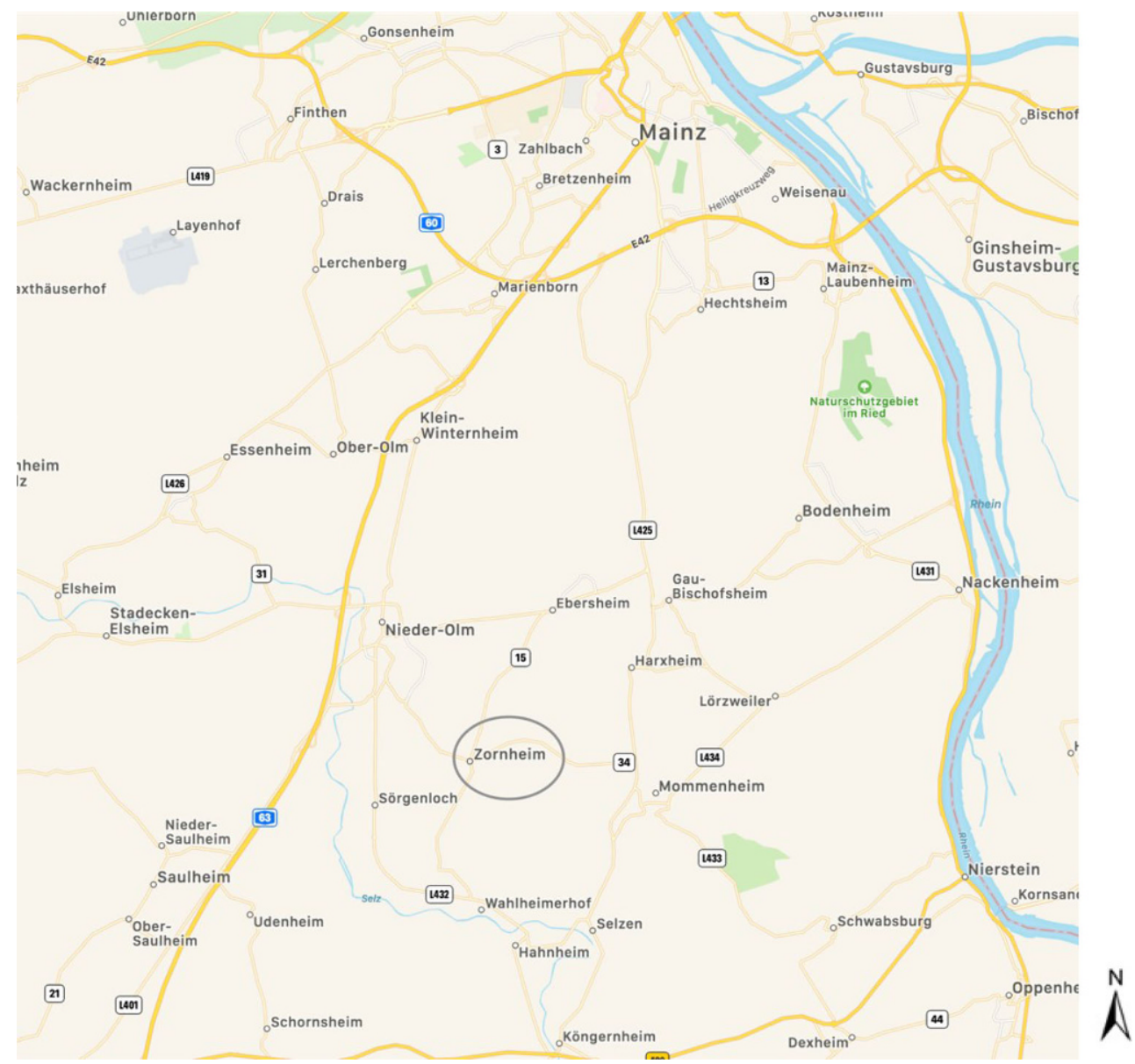

Als Referenzfläche dient eine im Gebiet liegende, 1995 gepflanzte Streuobstwiese (Abb. 3e) mit Apfel-, Birnen-, Mirabellen-, Kirsch- und Zwetschenbäumen. Der Unterwuchs wächst im Laufe der Vegetationsperiode hoch auf und wird im Herbst einmal gemäht.

Die Erwerbsobstanlagen werden von den Landwirten nach wirtschaftlichen Gesichtspunkten im Rahmen der Integrierten Produktion (IP) behandelt. Die Referenzfläche Streuobst wird nicht wirtschaftlich genutzt und einmal im Jahr gemäht.

\section{Material und Methoden}

\section{Datenerfassung}

Um möglichst viele der in den Erwerbsobstanlagen lebenden Insekten und Spinnentiere zu erfassen, wurden verschiedene Fangtechniken angewandt (Probenahme: 26.VI.-31.VIII.2016, und 13.IV.-28.VI.2017). Zur Erfassung der in den Anlagen fliegenden Tiere wurde in jeder Anlage eine Flugfalle (Lufteklektor nach Rahn) in der Mitte der Anlage zwischen zwei Bäumen in ca. zwei Metern Höhe aufgehängt, so dass die Fangfläche (zwei gekreuzte Plexiglas-Prallscheiben) auf einer ungefähren Höhe von $120-150 \mathrm{~cm}$ hing. Die Fangflasche wurde zur Hälfte mit Fangflüssigkeit (gesättigte NaCl-Lösung + ein Tropfen Handspülmittel als Detergenz) gefüllt, die Fallen 14-tägig gewechselt. Die Proben wurden im Labor entsalzt und dann in Ethanol (70\%) überführt.

Zur Erfassung der Bodenfauna wurden in jeder Anlage fünf Bodenfallen (Honiggläser, $500 \mathrm{ml}$ ) im Herbizidstreifen der Baumreihe mit der Flugfalle im Abstand von ca. zehn Metern ausgebracht. Die Gläser wurden ebenerdig eingegraben und zur Hälfte mit Fangflüssigkeit gefüllt. Auf der Streuobstwiese wurden die Fallen in einer geraden Linie zwischen zwei entfernt stehenden Bäumen eingegraben und mit einer umgedrehten Petrischale überdacht, um das Hereinfallen von Wirbeltieren (Mäusen, Kröten, Eidechsen) zu verhindern. Die Bodenfallen wurden während der Fangzeit alle 14 Tage gewechselt. Die Tiere wurden im Labor wie die Fänge aus den Flugfallen weiterbearbeitet.

Alle 14 Tage wurden auf den Flächen Klopfproben genommen. Hierfür wurden auf jeder Fläche 100 Äste geklopft, wobei mit einem gepolsterten Klopfstock je dreimal in schneller Folge auf den Ast geschlagen wird. Die herabfallenden Tiere wurden mit einem Klopfschirm aufgefangen, durch den angebrachten Trichter in einen Gefrierbeutel 
Abb. 2 Luftbild Zornheim mit Untersuchungsflächen. (Quelle: LANIS RLP 2020)

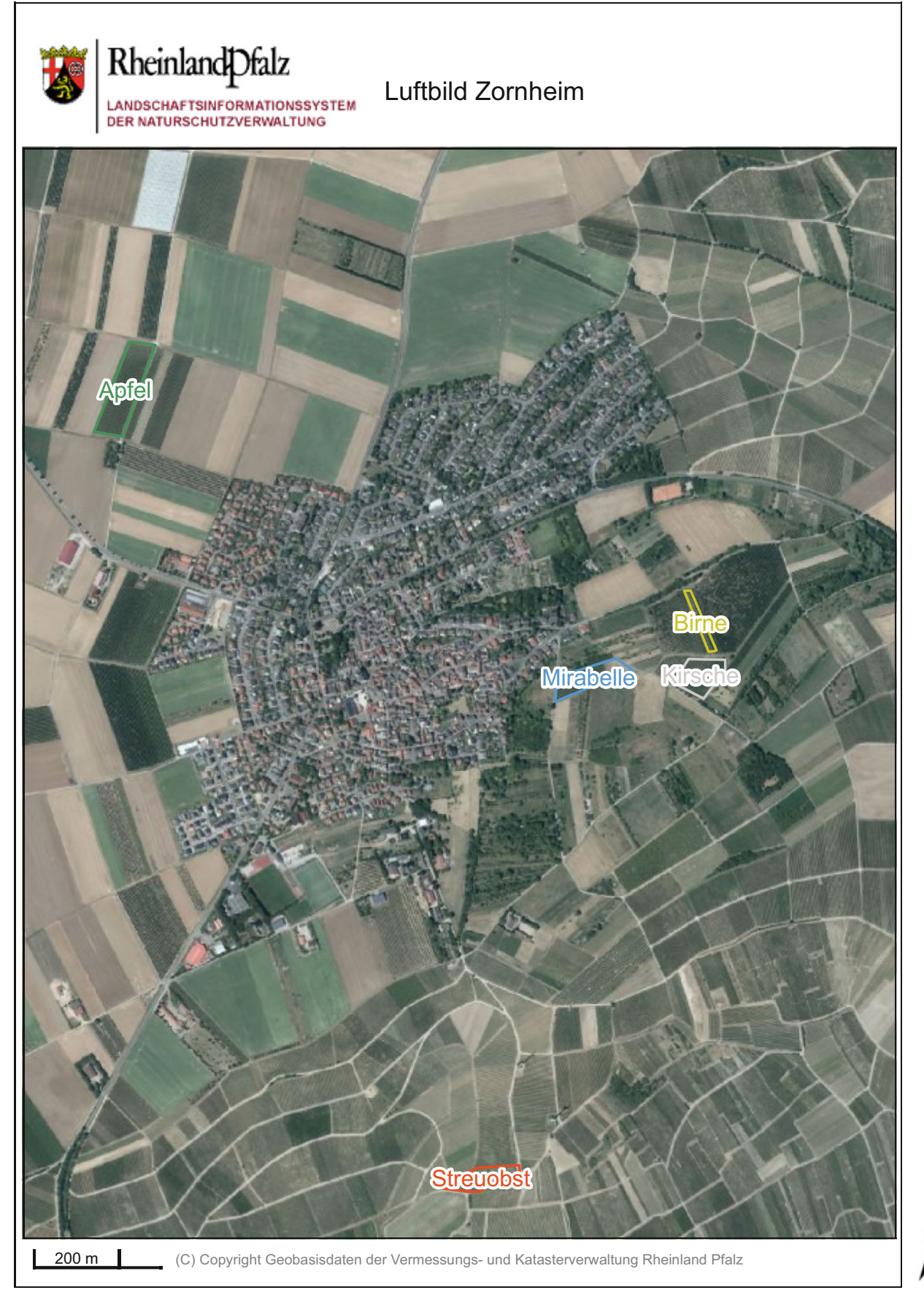

$\bigwedge^{N}$ geleitet, im Labor tiefgefroren und später wie die anderen Proben weiterbearbeitet.

Um die Auswirkungen von Bewirtschaftung und Strukturen auf die Fauna zu ermitteln, wurden die Mahd- und Pflanzenschutzmitteleinsätze der Landwirte anhand der Betriebshefte erfasst. Die Strukturen wurden mittels eines im Projekt erarbeiteten Erfassungsbogens aufgenommen.

\section{Auswertung der Biodiversität der Insekten und Spinnentiere}

Die Proben wurden nach Großgruppen sortiert und ausgezählt. Die auf Ordnungsniveau zugeordneten Tiere wurden tabellarisch für jede Untersuchungsfläche erfasst. Wegen der großen ökologischen Unterschiede wurden die Hautflügler (Hymenoptera) nicht gesamt betrachtet, sondern in Ameisen (Formicidae) und ,andere Hymenoptera“ unterteilt. Zur Ermittlung der Ähnlichkeit zweier Flächen wurde die Dominanzidentität $\left(I_{D}\right)$ der Großgruppen nach Ren- 
Abb. 3 Untersuchte Obstanlagen (oben) und Streuobstwiese (e, unten) im Umfeld von Zornheim (Rheinhessen). a Oben links Apfelanlage, b oben rechts Kirschanlage, c Mitte links Birnenanlage, d Mitte rechts Mirabellenanlage. (Fotos: Dannenmann)
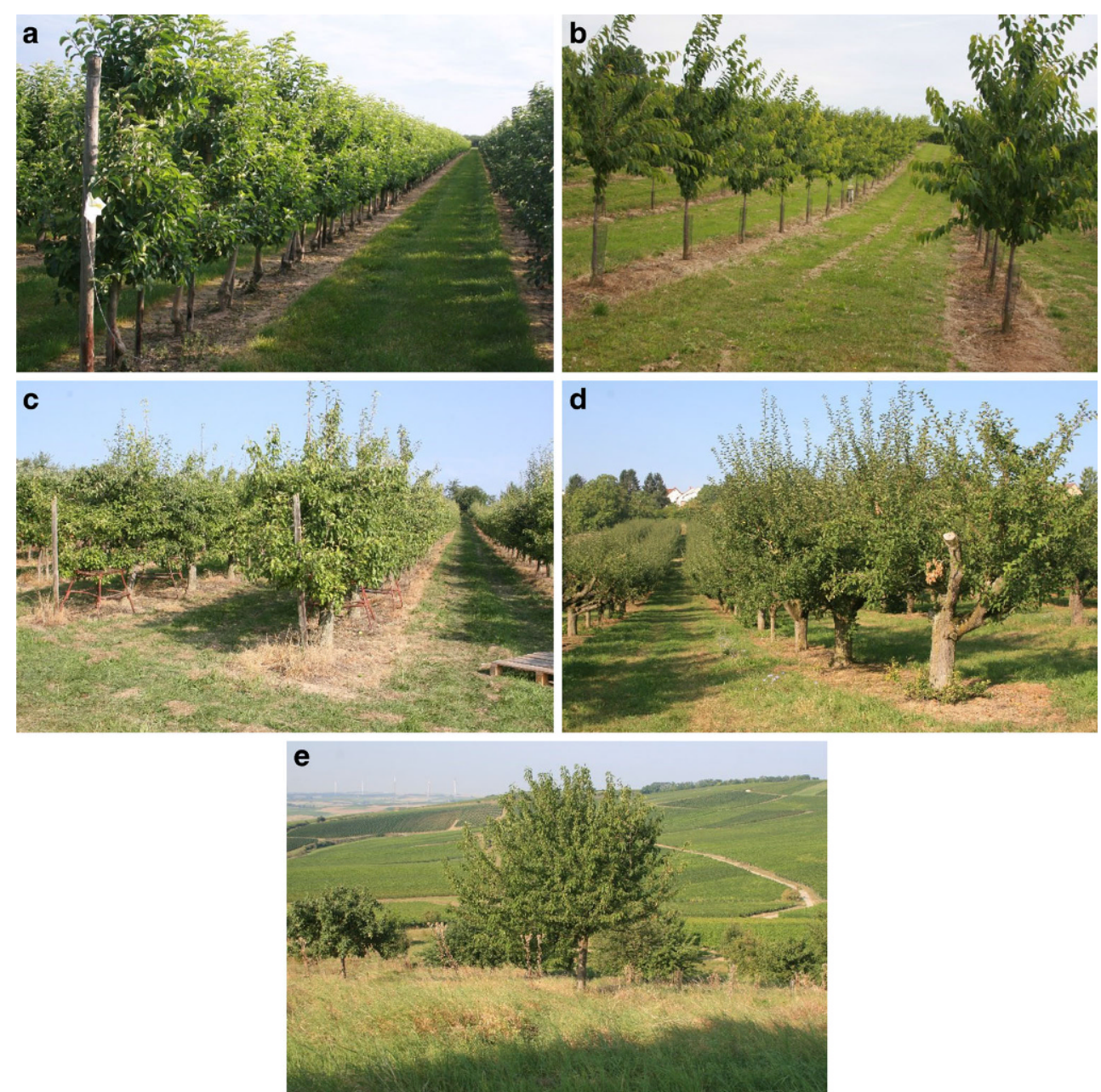

konen im Vergleich der einzelnen Erwerbsobstflächen mit der Streuobstwiese errechnet. Eine hohe $\mathrm{I}_{\mathrm{D}}$ zeigt eine starke Ähnlichkeit der Gruppendominanzen. Die Ähnlichkeiten der Artenverteilung wurden mittels Sörensen-Index (Maß für Übereinstimmung der Artenkombinationen verschiedener Artengemeinschaften) untersucht.

Für die auf Artniveau bestimmten Käfer (Coleoptera) und Wanzen (Heteroptera) wurde zusätzlich die Diversität nach Shannon $\mathrm{H}_{\mathrm{S}}$ mit Evenness $\mathrm{E}$ berechnet. Für diese Gruppen sind bzw. werden detaillierte Artenlisten mit faunistisch-ökologischen Angaben in der Folge publiziert (Wanzen: Dannenmann et al. 2019; Käfer: Dannenmann und Wagner 2020). Eine Belegsammlung der Käfer befindet sich im Naturhistorischen Museum in Mainz.

Die Diversität $\mathrm{H}_{\mathrm{S}}$ gibt die Mannigfaltigkeit einer Lebensgemeinschaft wieder. Sie steigt mit zunehmender Artenzahl und zunehmender Gleichverteilung der relativen Individuendichte (Anzahl der Individuen pro Fläche oder Probe) der einzelnen Arten an. Den maximalen Wert $\mathrm{H}_{\max }$ erreicht $\mathrm{H}_{\mathrm{S}}$ bei gleicher Individuendichte aller Arten (Bick 1989). Die Evenness (E) gibt das Verhältnis von vorhandener zu größtmöglicher Diversität der Artenzahl wieder. Sie ist ein $\mathrm{Ma} ß$ der Gleichverteilung der Individuen auf die Arten bei einer bestimmten Diversität (Schaefer 2012).

\section{Erfassung von Einflussfaktoren der Biodiversität}

Die Biodiversität von Obstanlagen kann durch strukturelle Gegebenheiten (Umland-, Anlagen-, Sonderstrukturen), durch Standortbedingungen sowie durch Bewirtschaftungsmaßnahmen beeinflusst werden. Die Strukturen des Umlandes wurden in $500 \mathrm{~m}$ Umkreis der Untersuchungsflächen auf Grundlage der Biotoptypenkartieranleitung von Rheinland-Pfalz (LANIS 2013) erfasst und ausgewertet (Heinrichs 2017). Die ermittelten Biotoptypen wurden nach der Biotoptypenbewertung Baden-Württemberg (LUBW 2005; für RLP nichts Vergleichbares vorhanden) mit ihrem prozentualen Anteil am Umland der jeweiligen Anlage bewertet und ihr Einfluss mit dem Pearson-Korrelationskoeffizienten ermittelt. Es konnte kein großer Zusammenhang nachgewiesen werden. Da das Umland nicht von dem einzelnen Bewirtschafter beeinflusst werden kann, wurde es nicht in den Bewertungsbogen aufgenommen, könnte aber bei der Erstaufnahme der Flächen vor Maßnahmen einen 
Tab. 1 Anzahl und Vergleiche der auf den Untersuchungsflächen nachgewiesenen Insekten und Spinnentiere

\begin{tabular}{llllll}
\hline $\begin{array}{l}\text { Untersuchungs- } \\
\text { fläche }\end{array}$ & $\mathrm{P}_{\mathrm{B}}$ & $\begin{array}{l}\text { Individuenzahl gesamt (Insekten } \\
\text { und Spinnentiere) }\end{array}$ & $\begin{array}{l}\text { Webspinnen (Araneae) } \\
\text { Individuenzahl }\end{array}$ & $\begin{array}{l}\text { Käfer (Coleoptera) } \\
\text { Individuenzahl }\end{array}$ & $\begin{array}{l}\text { Wanzen (Heteroptera) } \\
\text { Individuenzahl }\end{array}$ \\
\hline Streuobstwiese & 423 & 25.887 & 2996 & 1952 & 266 \\
Kirschanlage & 275 & 16.750 & 2837 & 1607 & 64 \\
Mirabellenanlage & 233 & 11.734 & 1529 & 704 & 32 \\
Birnenanlage & 229 & 13.715 & 1062 & 978 & 40 \\
Apfelanlage & 147 & 13.798 & 454 & 711 & 26 \\
\hline
\end{tabular}

$P_{B}$ erreichte Biodiversitätspunkte

Einfluss auf den Ausgangswert der Anlage haben. Dieses gilt ebenso für die Anlagengröße. Kleinere Anlagen könnten größere Randeffekte aufweisen.

Es wurden Sonderstrukturen wie Nisthilfen für Vögel und Insekten, Totholz, natürliche Höhlen, Gehölze und Blühstreifen in den Anlagen und direkt an die Anlagen angrenzend aufgenommen. Die Strukturaufnahme innerhalb der Anlage erfolgte mit den Schwerpunkten auf die Struktur der Bäume und ihrer Pflanzdichte (= Baumdichte). Um das Lebensraumangebot für Tiere zu ermitteln, wurden das Alter der Bäume, Baumhöhe und Stammumfang, der Anteil der Bäume mit Totholz sowie die Größe des Baumstreifens erfasst (Werner 2017). Die Standortfaktoren Sonne, Wind und Feuchte mit Einfluss auf die Biodiversität wurden über die Beschattung erfasst. Der Vergleich der Exposition ergab keine nennenswerten Unterschiede zwischen den Flächen (Heinrichs 2017), so dass diese als Standortfaktor nicht weiter einbezogen wurde. Als Bewirtschaf- tungsfaktoren wurden Mahd/Mulchen sowie der Einsatz von Pflanzenschutzmitteln (PSM) berücksichtigt.

\section{Bewertung der Einflussfaktoren der Biodiversität}

Um die Wirkung der verschiedenen Einflussfaktoren vergleichbar zu machen, wurde ein gewichteter Bewertungskatalog und auf dessen Grundlage ein Bewertungsbogen (Dannenmann et al. 2020) zur Erfassung der Einflussfaktoren erstellt. Über die vergebenen Punkte lässt sich eine Aussage über die wahrscheinlich vorhandene Biodiversität auf der Fläche und deren mögliche Aufwertung machen. In diesem Bewertungskatalog wurden für die Arthropoden wichtige Einflussfaktoren wie Sonderstrukturen in und am Rand der Anlage, Anlagenstruktur (Alter und Größe der Bäume, Baumdichte, Totholz, Anteil offener Fläche/Baumstreifen), Beschattung durch die Kronen und die Bewirtschaftungs-

Tab. 2 Vorkommen und Diversität der nachgewiesenen Käfer- und Wanzenarten

\begin{tabular}{|c|c|c|c|c|c|c|}
\hline \multirow[t]{2}{*}{ Untersuchungsfläche } & \multicolumn{3}{|c|}{ Käfer (Coleoptera) } & \multicolumn{3}{|c|}{ Wanzen (Heteroptera) } \\
\hline & Artenanzahl & $\mathrm{H}_{\mathrm{S}}$ & $\mathrm{E}$ & Artenanzahl & $\mathrm{Hs}_{\mathrm{s}}$ & $\mathrm{E}$ \\
\hline Streuobstwiese & 237 & 4,43 & 0,81 & 38 & 2,76 & 0,76 \\
\hline Kirschanlage & 203 & 4,37 & 0,82 & 24 & 2,70 & 0,85 \\
\hline Mirabellenanlage & 150 & 4,01 & 0,80 & 19 & 2,35 & 0,83 \\
\hline Birnenanlage & 127 & 3,56 & 0,73 & 20 & 2,49 & 0,83 \\
\hline Apfelanlage & 133 & 4,15 & 0,85 & 10 & 1,87 & 0,81 \\
\hline
\end{tabular}

$H_{S}$ Diversität nach Shannon, $E$ Evenness

Abb. 4 Korrelationskoeffizient Bewertungspunkte/Arten- und Individuenzahlen (nach Bravis \& Pearson), RL-Arten: Arten der Roten Liste (Geiser 1998)

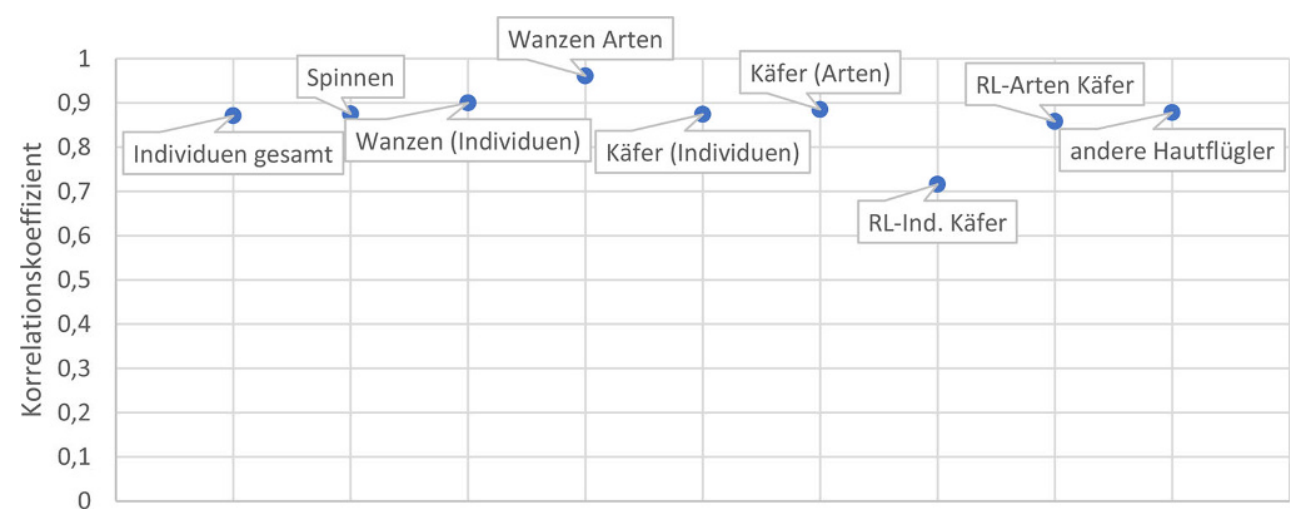


Tab. 3 Vorkommen von Käferarten der Roten Liste (Geiser 1998) und Individuenverteilung auf ausgewählte Großgruppen in den unterschiedlichen Fallen (Straten)

\begin{tabular}{|c|c|c|c|c|c|}
\hline \multirow{2}{*}{$\begin{array}{l}\text { Untersuchungs- } \\
\text { fläche }\end{array}$} & \multicolumn{5}{|c|}{ Coleoptera (Käfer) Artenzahl der Roten Liste 98} \\
\hline & $\begin{array}{l}\text { Vom Aussterben be- } \\
\text { droht (1) }\end{array}$ & Stark gefährdet (2) & Gefährdet (3) & Vorwarnliste (V) & $\begin{array}{l}\text { Geographisch sehr sel- } \\
\text { ten }(\mathrm{R})\end{array}$ \\
\hline Streuobstwiese & 1 & 8 & 13 & 4 & 1 \\
\hline Kirschanlage & 1 & 5 & 9 & 5 & 2 \\
\hline Mirabellenanlage & 2 & 4 & 6 & 4 & 1 \\
\hline Birnenanlage & 0 & 1 & 7 & 4 & 1 \\
\hline Apfelanlage & 0 & 5 & 5 & 5 & 1 \\
\hline
\end{tabular}
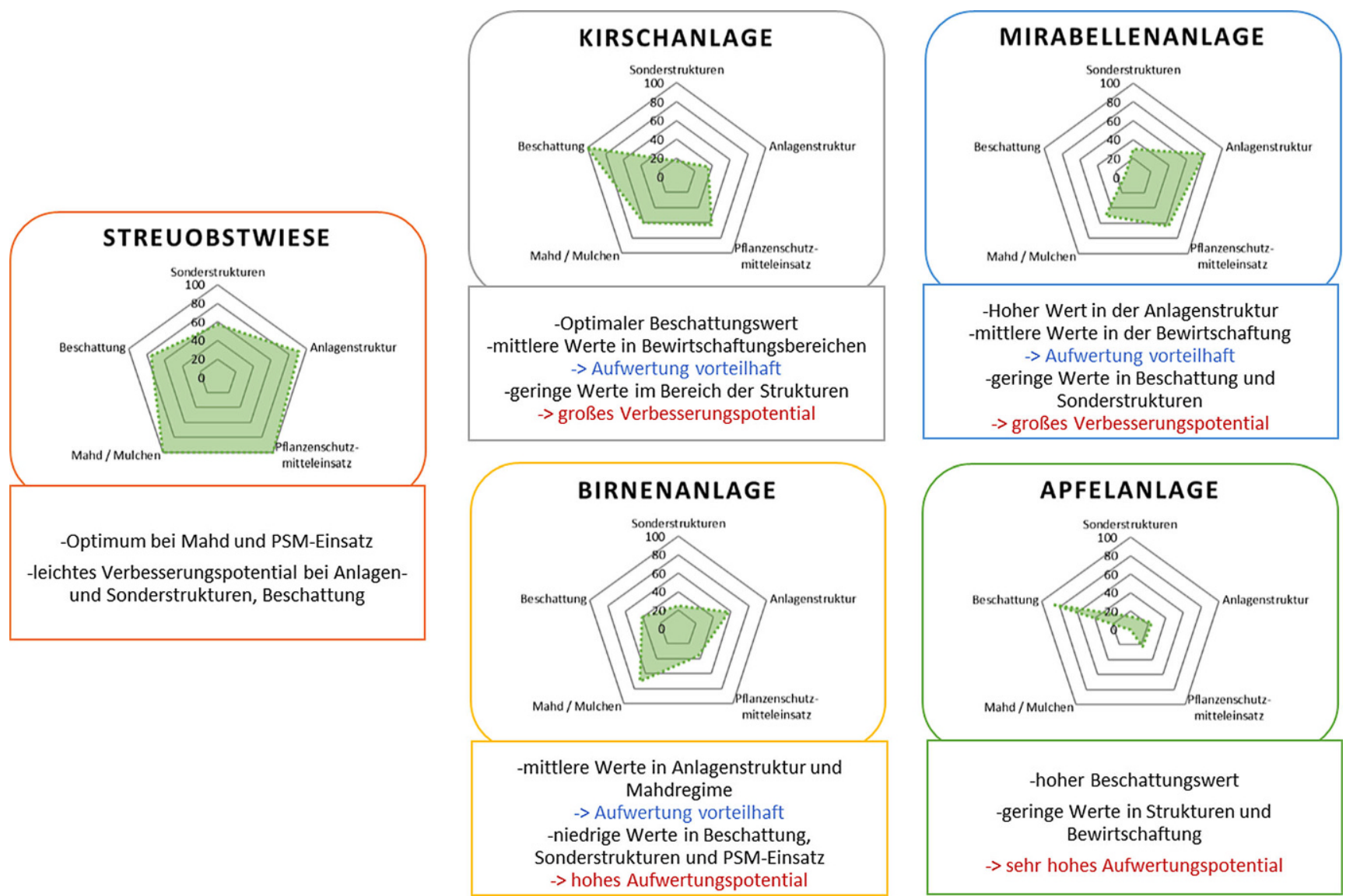

Abb. 5 Darstellung der in den einzelnen Bereichen erreichten Bewertungspunkte im Netzdiagramm mit Deckungsfläche (grün), Beschreibung des Aufwertungspotentials

intensität (Mulchgänge, PSM-Applikation) berücksichtigt. Für jede Fläche wurde so ein Punktwert ermittelt und dieser mit den gefundenen Arten- und Individuenzahlen verglichen. In Tab. 1 sind die anhand der Strukturen erreichten Biodiversitätspunkte $\left(\mathrm{P}_{\mathrm{B}}\right)$ im Vergleich zu den Individuenzahlen der auf den Flächen gefangenen Insekten und Spinnentiere dargestellt.

Tab. 2 zeigt Anzahl und Diversität der auf den verschiedenen Flächen nachgewiesenen Käfer- und Wanzenarten.

In Tab. 3 ist das Vorkommen von Käferarten der Roten Liste (Geiser 1998) in den untersuchten Anlagen dargestellt.
Es ließ sich ein hoher statistischer Zusammenhang zwischen dem erreichten Punktwert und der auf den Flächen gefundenen Biodiversität der Arthropoden (Abb. 4) zeigen (Korrelation nach Bravis und Pearson [Excel], Auswertung nach Duller 2019).

Die Übertragung der ermittelten Biodiversitäts-Punktwerte in Netzdiagramme zeigt deutlich, in welchen Bereichen Defizite vorhanden und Aufwertungen vorteilhaft sind. Die Darstellung im Netzdiagram (Abb. 5) zeigt für die Flächen große Differenzen in den Deckungsflächen mit deutlichen Unterschieden bei den Einflussfaktoren. Die größte Deckungsfläche (grün) zeigt wie erwartet die Referenzflä- 
che Streuobst. Da sie nicht bewirtschaftet wird, finden außer der jährlichen Mahd keine Eingriffe statt. Sie erreicht den optimalen Wert von $100 \%$ nicht, da in und am Rand der Anlage wenig Sonderstrukturen (Höhlen, Totholz) vorhanden und Stämme und Kronen für Streuobst eher klein sind. Die Kirschanlage erreicht trotz Nutzung eine relativ große Deckungsfläche mit 275 Biodiversitätspunkten $\left(\mathrm{B}_{\mathrm{P}}\right)$. Im Netzdiagramm werden die einschränkenden Faktoren deutlich: Anlagen- und Sonderstrukturpunkte erreichen nur geringe Werte, während die geringe Anzahl der Mulchgänge und PSM-Applikationen ebenso wie der Beschattungsanteil positiv auf die Arthropodenfauna wirken. In der Mirabellenund Birnenanlage sind $\mathrm{P}_{\mathrm{B}}$ und Größe der Deckungsfläche ähnlich, die Einflussfaktoren unterscheiden sich aber erheblich. Die Mirabellenanlage weist mit ihren natürlichen Höhlen und Totholz am Baum viele Anlagenpunkte auf. Die relativ geringe Intensität der Bewirtschaftung (wenig Mulchgänge, PSM-Einsatz) führt zu mittleren Punktwerten in diesen Bereichen. Nachteilig wirken sich die intensive Beschattung und wenige Sonderstrukturen aus (starke Einschnitte der grünen Fläche). Die Birnenanlage erreicht durch wenige Mulchgänge und Anlagenstrukturen Punkte über $50 \%$, wenige Sonderstrukturen, starke Beschattung und PSM-Einsatz schränken hier die Werte ein. In der intensiv gepflegten Apfelanlage (geringster $\mathrm{P}_{\mathrm{B}}$-Wert) gibt es kaum Sonderstrukturen und wenig Anlagenstrukturen. Starker PSM-Einsatz und häufiges Mulchen führen zu starken

Tab. 4 Maßnahmen zur Erhöhung des Sonderstrukturwertes

\begin{tabular}{ll}
\hline Maßnahme & Wirkung \\
\hline Erhalt und Pflanzung & Schaffung von Lebens- und Rückzugs- \\
großer Einzelbäume & raum für viele Insekten, Spinnentiere, \\
$(>5 \mathrm{~m}$, Abb. 6), Sträucher & Vögel und andere Wirbeltiere \\
$(>1,5 \mathrm{~m})$ und Hecken am &
\end{tabular}

Rand der Anlage

Einbringen von geeigneten Nisthilfen (,Wildbienenhotel“", Vogelnistkästen)

Anlage und Erhalt von Blühzonen in und am Rand der Anlage (Abb. 7)

Überjähriger Erhalt von höherer Randvegetation, Hochstauden und Altgrassäumen mit markhaltigen und hohlen Stängeln

Zulassen natürlicher Höhlenbildung (Abb. 8)

Anlage von Totholzhaufen
Förderung von Wildbienen und anderen Insekten (Brutraum und Winterquartier)

Nahrung für die auf Pollen (Larvenfutter Wildbienen) und Nektar (z. B. bienen, Florfliegen, Käfern) angewiesenen Insekten, Pflanzenlebensraum

Nistplatz und Winterquartier für halmbrütende Insekten (Käfer, Wildbienen, Überwinterung als Imago, Ei, Larve oder Puppe möglich), neue Generation wird durch Mahd vernichtet

Nistplatz und Winterquartier für Insekten (z. B. Prachtkäfer, Holzbienen), Winterquartier und Lebensraum für weitere Tiere wie Vögel, Bilche (Schläfer), Fledermäuse

Nistplatz und Winterquartier, z. B. Steinbienen Imagines von Schmetterlingen, Wild-
Einschränkungen, positiv wirkt nur der Beschattungsgrad. Nur hier werden über 50 Punkte erreicht.

Eine ausführliche Beschreibung der Bewertungsmethode von Obstanbauflächen auf Basis der Arthropodengruppen wurde in Naturschutz und Landschaftsplanung vorgestellt (Dannenmann et al. 2020). Die Wanzenfauna wurde bereits veröffentlicht (Dannenmann et al. 2019), eine Publikation über die Diversität der Käfer in Erwerbsobstanlagen befindet sich im Druck (Dannenmann et al.).

\section{Ableitung von biodiversitätsfördernden Maßnahmen}

Die faunistischen Unterschiede lassen sich auf verschiedene Faktoren zurückführen. Es konnte gezeigt werden, dass die Strukturen und die Bewirtschaftung über Bereitstellung oder Einschränkung von Lebensräumen einen großen Einfluss auf das Vorkommen und die Verteilung der Insekten und Spinnentiere haben. Über den Bewertungsbogen (Dannenmann et al. 2020) lässt sich für vorhandene Obstanlagen praxisorientiert und ohne größeren Arbeitsaufwand identifizieren, in welchen Bereichen (Anlagen-, Sonder-,

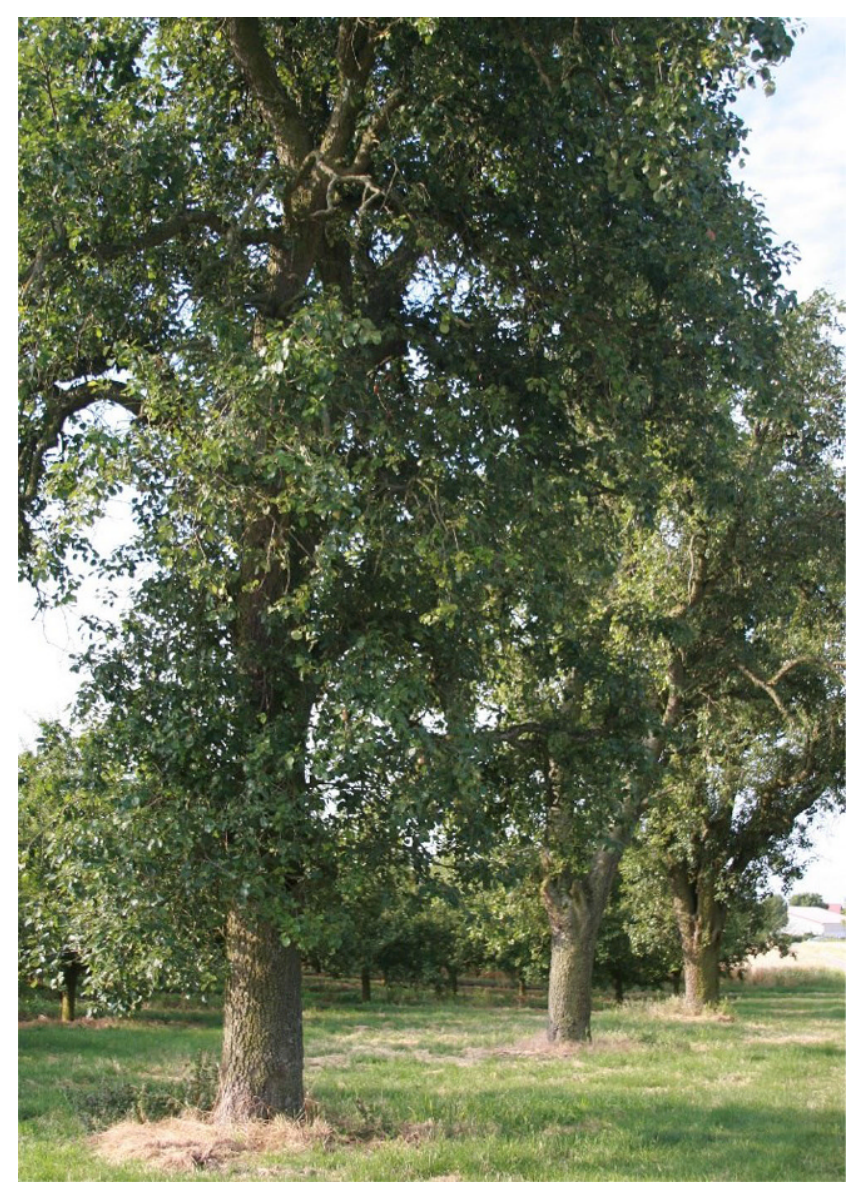

Abb. 6 Alte Birnbäume. (Foto Dannenmann) 


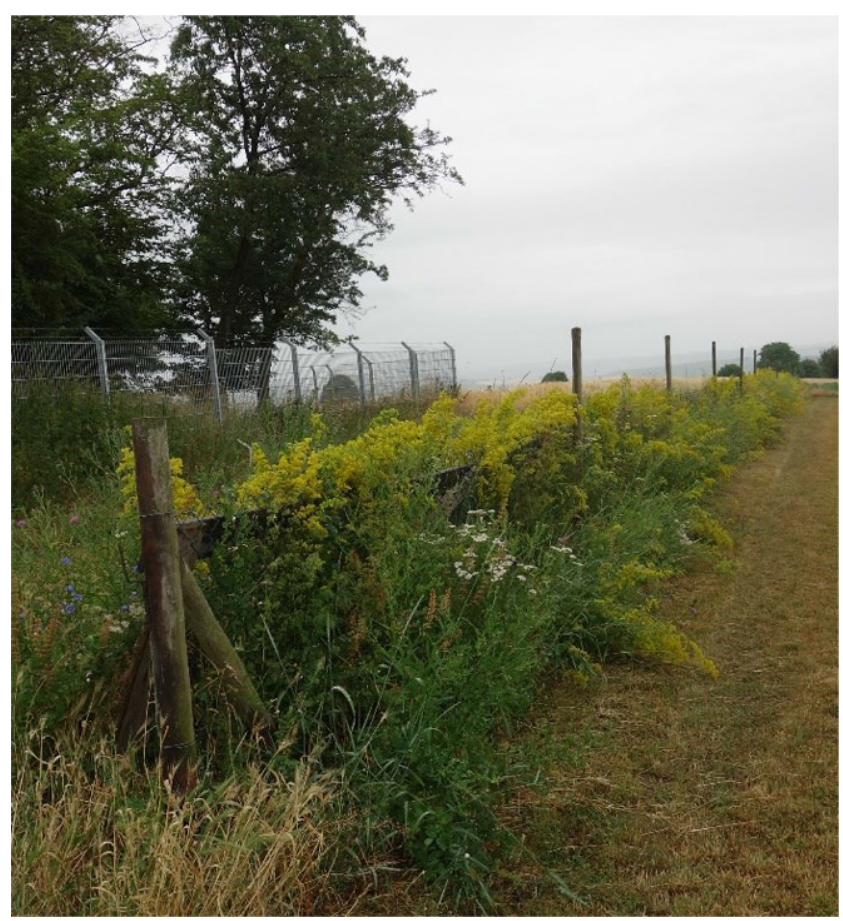

Abb. 7 Blühstreifen am Zaun. (Foto: Dannenmann)

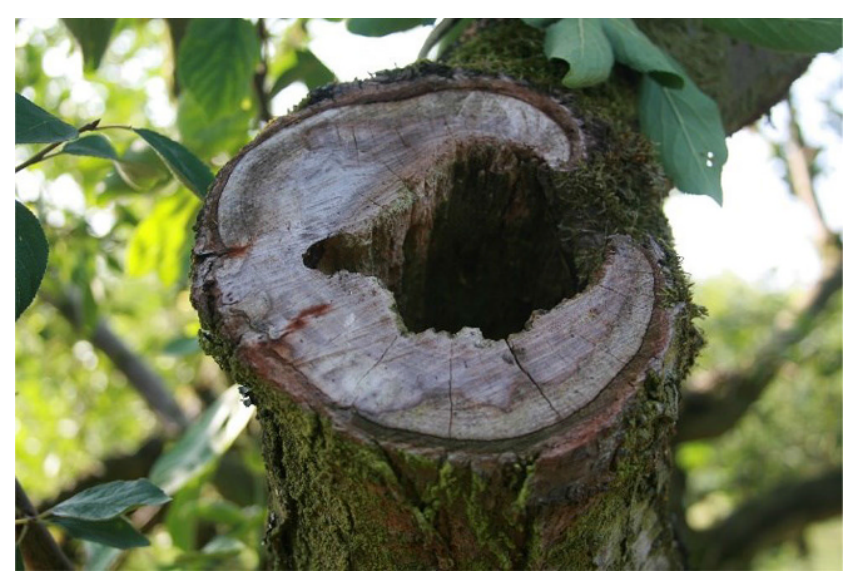

Abb. 8 Natürliche Höhle. (Foto: Dannenmann)

Baumstrukturen (Beschattung), Bewirtschaftung) Maßnahmen sinnvoll sind, um die Biodiversität zu erhöhen. Die in Zornheim gewonnenen Erkenntnisse werden durch weitere Untersuchungen in ergänzenden Projekten seit 2018 vervollständigt. Es zeigt sich auch dort, dass gezielte Maßnahmen zur Strukturförderung und Verminderung der Bewirtschaftungsintensität förderlich auf die Biodiversität von Insekten wirken.

Von den im Projekt erarbeiteten Ergebnissen wurden Maßnahmen abgeleitet, welche dazu beitragen sollen, dass auf den Erwerbsobstflächen gefundene große ökologische Potenzial zu einer biologischen Vielfalt auszubauen. Die im Folgenden dargestellten Maßnahmen sind wahlweise einzeln oder gesamt umzusetzen, angepasst an die jeweili-
Tab. 5 Maßnahmen zur Förderung der Anlagenstruktur

\begin{tabular}{ll}
\hline Maßnahme & Wirkung \\
\hline Belassen von Totholz am & Förderung von holznistenden Insek- \\
Baum (Abb. 9 und 10), & ten, wie Prachtkäfer, Hummeln und \\
stehend in der Anlage und & Steinbienen und Winterquartier (auch \\
am Rand (Kontrolle auf & für Wirbeltiere)
\end{tabular}

Schaderreger nötig)

Geringere Baumdichten, dadurch bessere Entfaltung der Einzelbäume, größere Kronen

Baumstreifen bis $1 / 3$ der Bodenfläche

Pflanzung stärker wachsender Unterlagen

Verlängerung der Umtriebszeit (>25 Jahre)

Teilerneuerung (Abb. 11) bei alten, abgängigen Anlagen (keine vollständige Rodung, z. B. je Jahr drei Reihen ersetzen)

Belassen von Mulm in oder am Rand der Anlage

Mehr Lebensraum und besseres Mikroklima für die von der Umgebungstemperatur und Feuchtigkeit abhängigen Arthropoden

Bietet positives Verhältnis von offenem Boden zu Grünland, fördert bodenlebende und dort nistende Insekten (z. B. Laufkäfer, Wildbienen) Größerer Stammumfang, stärkere Äste, mehr Holz, fördert Moos- und Flechtenbewuchs, potenziell mehr Lebensraum und Bruthöhlen für Insekten (Käfer, Spinnen, Raubwanzen) Ausbildung stabilerer Populationen in länger vorhandenem Lebensraum

Erhalt der vorhandenen Biodiversität im Altbestand, daraus schnellere Neubesiedelung der jungen Pflanzung

Winterquartier für Insekten (z. B. Marienkäfer)

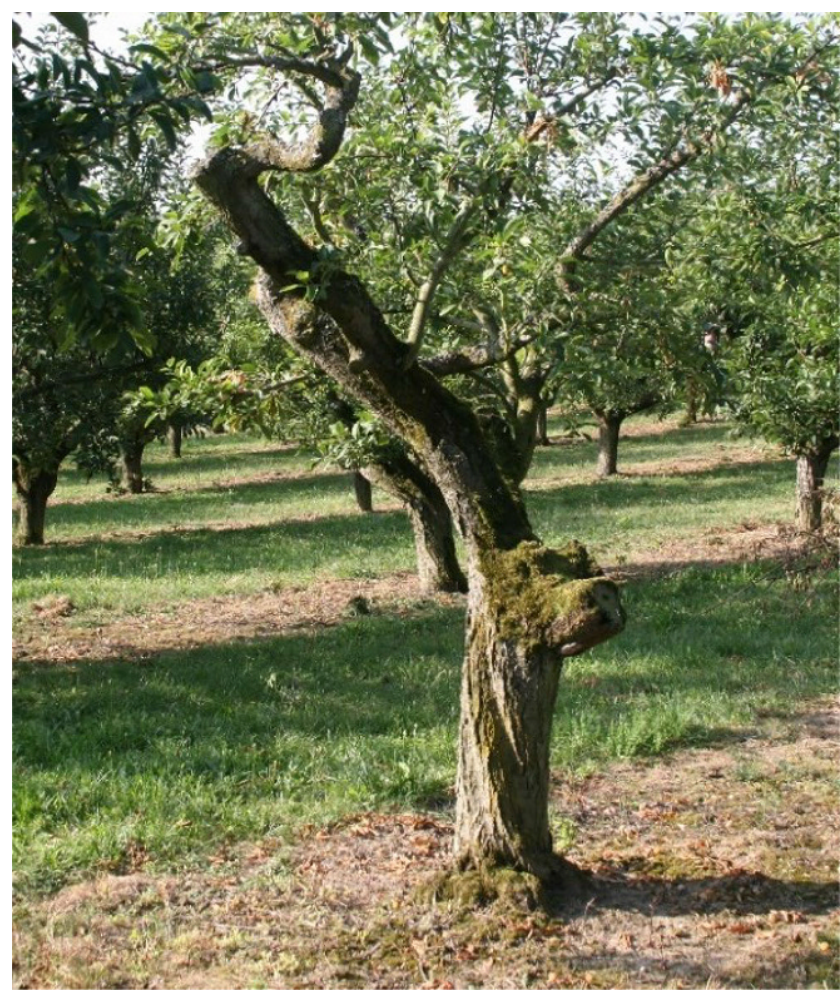

Abb. 9 Stehendes Totholz. (Foto: Dannenmann) 


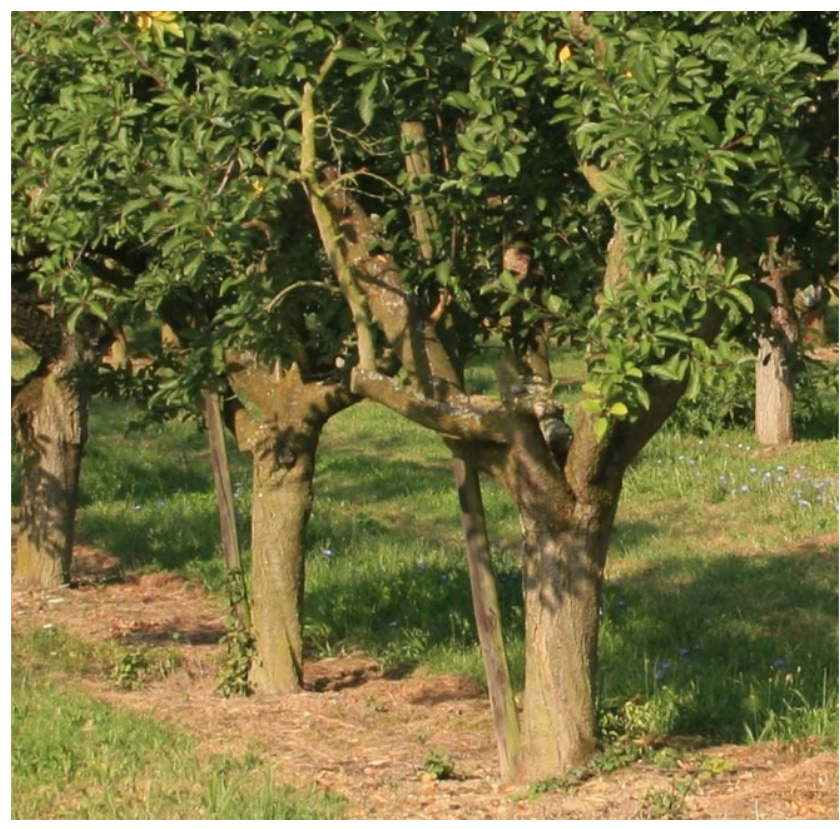

Abb. 10 Totholz am Baum. (Foto: Dannenmann)

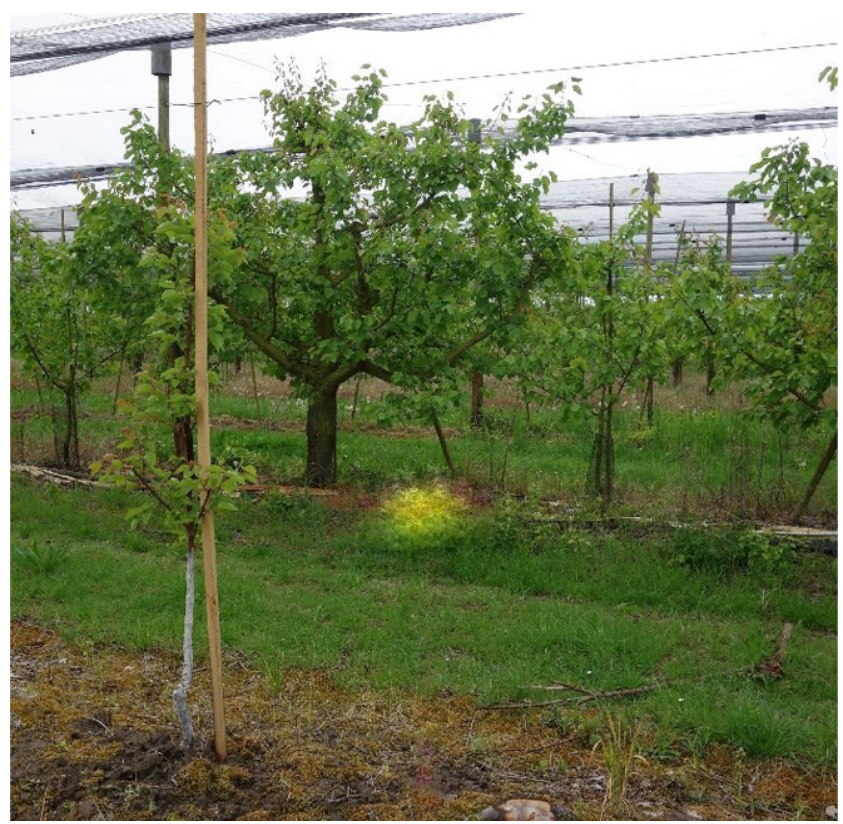

Abb. 11 Teilerneuerung. (Foto: Dannenmann)

gen Betriebsbedingungen. Die Einführung möglichst vieler Maßnahmen ist hierbei wünschenswert.

\section{Erhöhung des Sonderstrukturwertes}

Tab. 4 zeigt mögliche Maßnahmen zu Schaffung von Sonderstrukturen und des damit verbundenen Sonderstrukturwertes.

Beispiele (Abb. 6, 7 und 8)

\section{Erhöhung des Anlagenstrukturwertes}

In Tab. 5 werden Maßnahmen zur Förderung der Strukturen innerhalb der Anlage vorgeschlagen, um die Ausbildung stabiler Populationen zu fördern. Gerade Nützlingspopulationen profitieren hiervon.

Beispiele (Abb. 9, 10 und 11)

\section{Bewirtschaftung}

Mögliche Extensivierungsmaßnahmen zur Erhöhung der Biodiversität werden in Tab. 6 dargestellt. Negative Auswirkungen wie z.B. Ertragsminderung sollten kompensiert werden.

Beispiele (Abb. 12 und 13)

\section{Förderung der Biodiversität in der Kulturlandschaft allgemein}

Auch in der Kulturlandschaft außerhalb der Obstanlagen können gezielte Maßnahmen zur Erhöhung der Biodiversität, Stabilisierung der Populationen und Schaffung von

Tab. 6 Maßnahmen in der Bewirtschaftung

\begin{tabular}{ll}
\hline Maßnahme & Wirkung \\
\hline Verbesserung des Mahdregimes & Erhält Rückzugsraum und \\
(Abb. 12) durch: & Nahrung (Pollen, Nektar) in \\
Reduktion der Mulchgänge & Teilen der Fläche für Fauna \\
alternierendes Mulchen & Fördert Flora allgemein
\end{tabular}

Streifenmulchen

Möglichst geringer PSM-Einsatz: Geringere Einflussnahme auf nach Bedarf ausrichten (Kontrolle nötig) möglichst biologische und biotechNicht-Zielorganismen Stärkt Populationen insgesamt

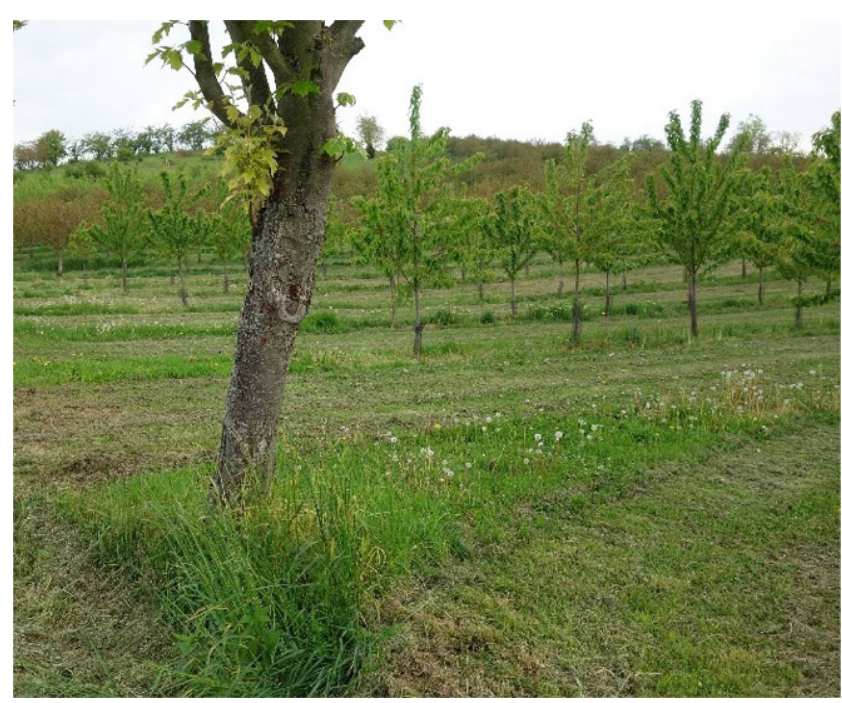

Abb. 12 Unvollständige Mahd, Reste des Blütenhorizontes bleiben stehen. (Foto Dannenmann) 


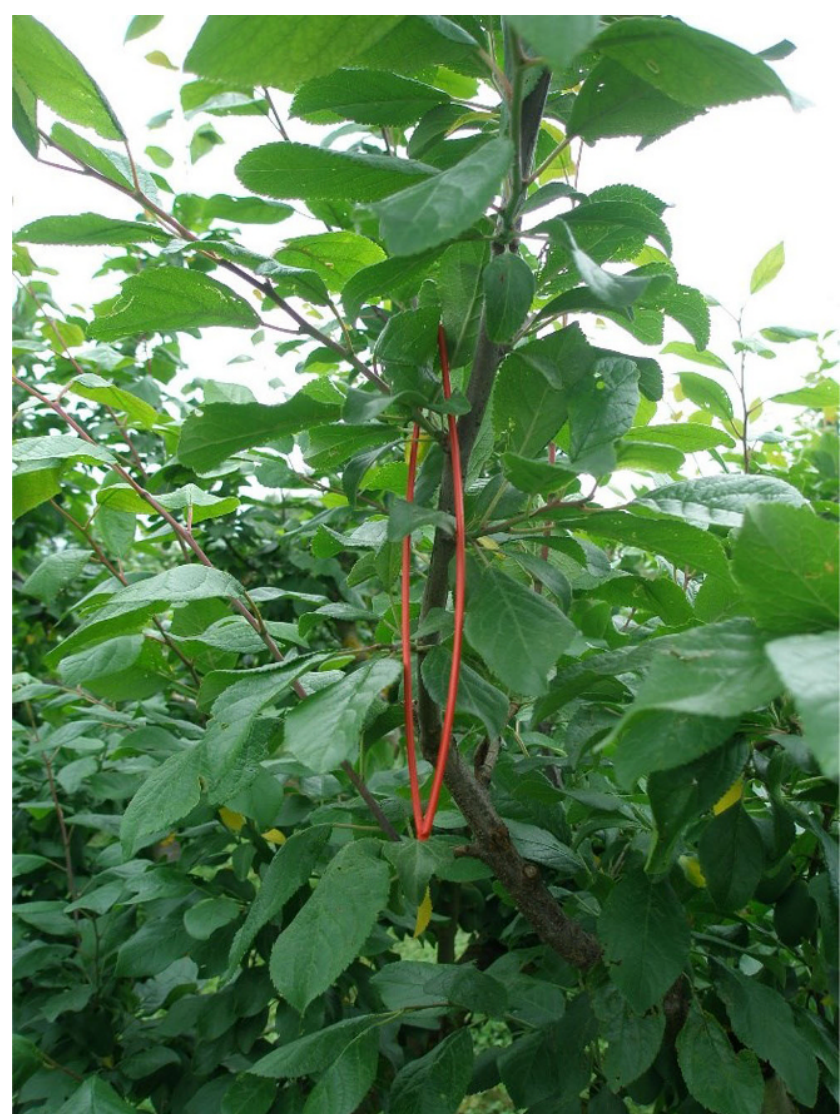

Abb. 13 Pheromondispenser Pflaumenwickler. (Foto: Harzer)

Tab. 7 Allgemeine Maßnahmen zur Förderung der Biodiversität in der Kulturlandschaft

\begin{tabular}{ll}
\hline Maßnahme & Wirkung \\
\hline Förderung und Erhalt unbe- & Bereitstellung von Nistraum \\
festigter Wege und anderer & (Abb. 14 und 15) für bodennistende \\
Bodenstellen mit wenig oder & Insekten (z. B. Laufkäfer, 70\% der \\
keiner Vegetation & heimischen Wildbienen) \\
Verhinderung des Verbra- & Erhalt der Lebensräume, Biodiver- \\
chens der Obstanlagen & sität nimmt mit fortschreitender \\
& Verbuschung ab \\
\hline
\end{tabular}

Rückzugsräumen führen. Tab. 7 stellt mögliche Maßnahmen dar.

Beispiele (Abb. 14 und 15)

\section{Fazit}

Insekten und andere Arthropoden brauchen Lebensräume, Nahrung und Nistplatz. Gerade die Insekten mit vollständiger Verwandlung, d.h. mit einer Entwicklung vom Ei über Larve und Puppe zu dem geschlechtsreifen Tier (Imago) bleiben die längste Zeit ihres Lebens im Larvenstadium, während das Imago nur kurze Zeit zur Fortpflanzung dient (Bsp. Junikäfer: Larvalzeit 2-3 Jahre, Flugzeit des Imagos

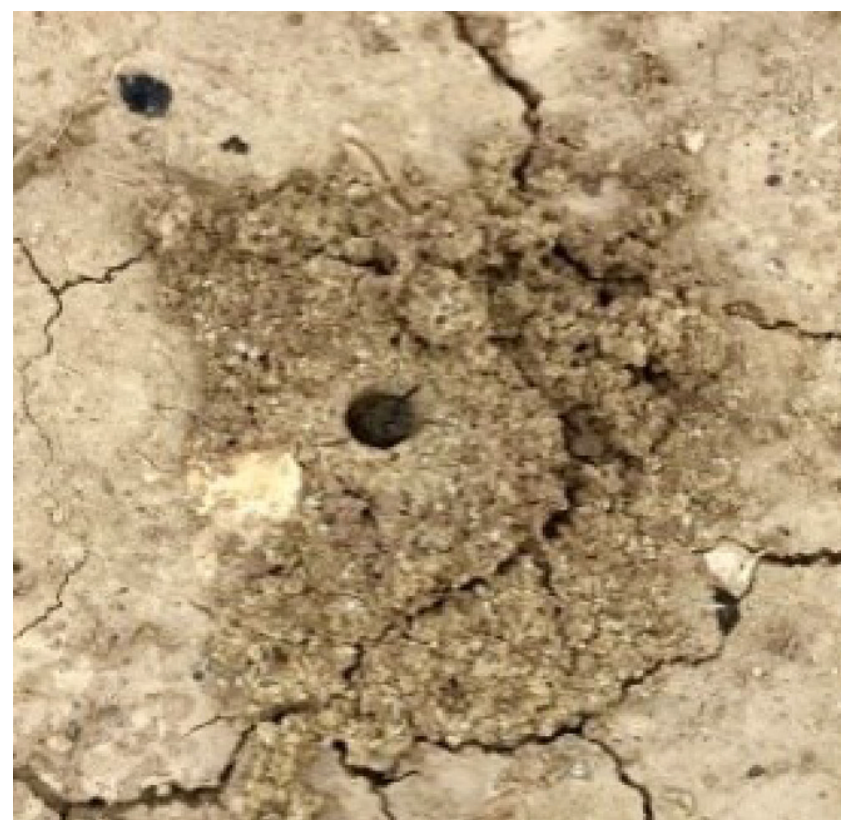

Abb. 14 Wildbienennest in unbefestigtem Fahrweg. (Foto Rest)

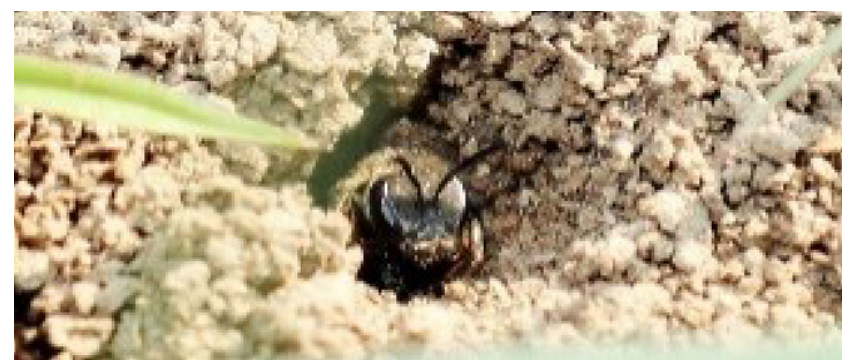

Abb. 15 Wildbiene im Nesteingang. (Foto Rest)

2-3 Monate). Der Einsatz von Insektiziden wirkt direkt auf die vorhandenen Tiere, während der Verlust von Nahrung und Nistplatz indirekt über längere Zeit zum Rückgang der Populationen führt. Die vorgeschlagenen Maßnahmen fördern über die Bereitstellung von Strukturen als Nistplatz, Überwinterungsquartier und Nahrungsangebot und verringerte Eingriffsintensität die Biodiversität der Insekten und Spinnentiere. Viele Insekten überwintern als Ei oder Larve im Boden oder Hohlräumen, wie z.B. Stängeln (z.B. Mauerbienen (Westrich 2018)). Werden im Herbst Ränder „sauber“ abgemäht, wird hiermit auch ein großer Teil der nächsten Generation vernichtet.

Von den vorgeschlagenen Maßnahmen führen einige evtl. zu Mindererträgen bzw. zu höheren Kosten. Diese sollten aus Naturschutzmitteln, wie z.B. Ersatzzahlungsmitteln, Ausgleichszahlungen durch die Gemeinden oder möglicherweise über AUKM ausgeglichen werden. Hiermit können die Populationen gestärkt und die Vielfalt der Insekten und Spinnentiere erhöht werden. 


\section{Dank}

Die der Publikation zu Grunde liegende Studie: „Honorierung von Landschaftspflegeleistungen im Erwerbsobstanbau“ wurde von der Stiftung Natur und Umwelt Rheinland-Pfalz gefördert. Wir danken dem Dienstleistungszentrum Ländlicher Raum Rheinhessen-Nahe, der Gemeinde Zornheim und den teilnehmenden Landwirten für die Bereitstellung von Daten.

Funding Open Access funding enabled and organized by Projekt DEAL.

Interessenkonflikt D. Dannenmann, E. Hietel und T. Wagner geben an, dass kein Interessenkonflikt besteht.

Open Access Dieser Artikel wird unter der Creative Commons Namensnennung 4.0 International Lizenz veröffentlicht, welche die Nutzung, Vervielfältigung, Bearbeitung, Verbreitung und Wiedergabe in jeglichem Medium und Format erlaubt, sofern Sie den/die ursprünglichen Autor(en) und die Quelle ordnungsgemäß nennen, einen Link zur Creative Commons Lizenz beifügen und angeben, ob Änderungen vorgenommen wurden.

Die in diesem Artikel enthaltenen Bilder und sonstiges Drittmaterial unterliegen ebenfalls der genannten Creative Commons Lizenz, sofern sich aus der Abbildungslegende nichts anderes ergibt. Sofern das betreffende Material nicht unter der genannten Creative Commons Lizenz steht und die betreffende Handlung nicht nach gesetzlichen Vorschriften erlaubt ist, ist für die oben aufgeführten Weiterverwendungen des Materials die Einwilligung des jeweiligen Rechteinhabers einzuholen.

Weitere Details zur Lizenz entnehmen Sie bitte der Lizenzinformation auf http://creativecommons.org/licenses/by/4.0/deed.de.

\section{Literatur}

Achtziger R, Nigmann U, Richert E, Scholze W (1999) Ökologische Untersuchungen zur Erfolgskontrolle und naturschutzfachlichen Bewertung von Streuobstbeständen - Durchführungskonzept und erste Ergebnisse. Schriftenreihe des Bayerischen Landesamtes für Umweltschutz Heft 150, Beiträge zum Artenschutz 22. Bayerisches Landesamt für Umweltschutz, Augsburg, S 227-243

Bick H (1989) Ökologie. Gustav Fischer, Stuttgart

Dannenmann D, Günther H, Wagner T (2019) Zur Wanzenfauna (Insecta: Heteroptera) konventionell genutzter Obstkulturen im Ver- gleich zu einer Streuobstwiese in Zornheim, Rheinhessen. Mainz Naturwiss Arch 56:249-258

Dannenmann D, Hietel E, Wagner T (2020) Insekten in der Kulturlandschaft. - Naturschutz und Landschaftsplanung. Eugen Ulmer Verlag, Stuttgart

Dannenmann D, Wagner T (2020) Zur Käferfauna (Insecta: Coleoptera) integriert bewirtschafteter Erwerbsobstanlagen im Vergleich zu einer Streuobstwiese in Zornheim, Rheinhessen. Mainzer Naturwissenschaftliches Archiv, Mainz (im Druck)

Duller C (2019) Einführung in die Statistik mit Excel und SPSS. Springer, Berlin, Heidelberg

Geiser R (1998) Rote Liste der Käfer Deutschlands. Schriftenreihe für Landschaftspflege und Naturschutz 55. Godesberg, Bonn-Bad, S $168-230$

Heinrichs D (2017) Biodiversität von Erwerbsobstanlagen - Untersuchung von Arthropodengesellschaften und abiotischen sowie anthropogenen Einflussfaktoren am Beispiel der Gemarkung Zornheim in Rheinhessen, Rheinland-Pfalz. Bachelorarbeit TH Bingen, unveröffentlicht. TH Bingen, Bingen

Landesamt für Umweltschutz und Gewerbeaufsicht Rheinland-Pfalz (1992) Begleituntersuchungen zum Biotopsicherungsprogramm „Streuobstwiesen“. Beiträge zur Landespflege in RheinlandPfalz, Bd. 15. Landesamt für Umweltschutz und Gewerbeaufsicht Rheinland-Pfalz (Hrsg.), Oppenheim

Landesanstalt für Umweltschutz Baden-Württemberg (2005) Bewertung der Biotoptypen Baden-Württembergs zur Bestimmung des Kompensationsbedarfs in der Eingriffsregelung. http://www. lubw.baden-wuerttemberg.de/documents/bewertung. Zugegriffen: 10. Okt. 2020

Landschaftsinformationssystem der Naturschutzverwaltung (LANIS) (2013) Biotope. http://www.naturschutz.rlp.de/?q=biotope. Zugegriffen: 19. Apr. 2017

Landschaftsinformationssystem der Naturschutzverwaltung (LANIS) (2020) Kartendienst. https://geodaten.naturschutz.rlp.de/karten dienste_naturschutz. Zugegriffen: 17 Dez 2020

Schaefer M (2012) Wörterbuch der Ökologie, 5. Aufl. Spektrum Akademischer Verlag, Heidelberg

Statistisches Landesamt RLP (2012) Statistische Berichte, Obstanbauerhebung 2012. Statistisches Landesamt RLP, Bad Ems

Statistisches Landesamt RLP (2017) Statistische Berichte 2017: Baumobstanbauerhebung - Flächen der Obstanlagen und Obstbaumbestände. Statistisches Landesamt RLP, Bad Ems

Werner R (2017) Untersuchung von floristischen und strukturellen Aspekten der Biodiversität in Erwerbsobstanlagen am Beispiel der Gemarkung Zornheim, Rheinhessen. Masterarbeit TH Bingen, unveröffentlicht. TH Bingen, Bingen

Westrich P (2018) Die Wildbienen Deutschlands. Ulmer, Stuttgart 